Pulsar Astronomy - 2000 and Beyond

ASP Conference Series, Vol. 202, 2000

M. Kramer, N. Wex, and R. Wielebinski, eds.

\title{
The evolution of helium white dwarfs: Applications for millisecond pulsars
}

\author{
T. Driebe and T. Blöcker \\ Max-Planck-Institut für Radioastronomie, Bonn, Germany \\ D. Schönberner
}

Astrophysikalisches Institut Potsdam, Potsdam, Germany

\section{White Dwarf Evolution}

Low-mass white dwarfs with helium cores (He-WDs) are known to result from mass loss and/or exchange events in binary systems where the donor is a low mass star evolving along the red giant branch (RGB). Therefore, He-WDs are common components in binary systems with either two white dwarfs or with a white dwarf and a millisecond pulsar (MSP). If the cooling behaviour of HeWDs is known from theoretical studies (see Driebe et al. 1998, and references therein) the ages of MSP systems can be calculated independently of the pulsar properties provided the He-WD mass is known from spectroscopy.

Driebe et al. $(1998,1999)$ investigated the evolution of He-WDs in the mass range $0.18<M_{\mathrm{WD}} / \mathrm{M}_{\odot}<0.45$ using the code of Blöcker (1995). The evolution of a $1 \mathrm{M}_{\odot}$-model was calculated up to the tip of the RGB. High mass loss terminated the RGB evolution at appropriate positions depending on the desired final white dwarf mass. When the model started to leave the RGB, mass loss was virtually switched off and the models evolved towards the white dwarf cooling branch. The applied procedure mimicks the mass transfer in binary systems. Contrary to the more massive $\mathrm{C} / \mathrm{O}-\mathrm{WDs}\left(M_{\mathrm{WD}} \geq 0.5 \mathrm{M}_{\odot}\right.$, carbon/oxygen core), whose progenitors have also evolved through the asymptotic giant branch phase, He-WDs can continue to burn hydrogen via the pp cycle along the cooling branch down to very low effective temperatures, resulting in cooling ages of the order of Gyr, i. e. of the same order of magnitude as the spin-down ages of millisecond pulsars. The mass-radius-relation for He-WDs shows significant evolutionary effects due to this residual nuclear burning. The ongoing hydrogen burning in He-WDs is provided by large envelope masses which decrease with increasing $M_{W D}$ (see Blöcker et al. 1997). In contrast to C/O-WDs, He-WDs cool down the slower the smaller their mass.

\section{Application to millisecond pulsars}

Based on their evolutionary models Driebe et al. (1998) determined the mass of the He-WD companion of the MSP PSR J1012+5307 (Nicastro et al., 1995, see also Sarna et al. 1998). They found $M_{\mathrm{WD}}=0.19 \pm 0.02 \mathrm{M}_{\odot}$ and $0.15 \pm 0.02 \mathrm{M}_{\odot}$ using the spectroscopic data of van Kerkwijk et al. (1996) and Callanan et al. (1998), resp. Thus, the mass ratio $M_{\text {pulsar }} / M_{\mathrm{He}-\mathrm{WD}} \approx 9.5 \pm 0.3$ (van Kerkwijk 


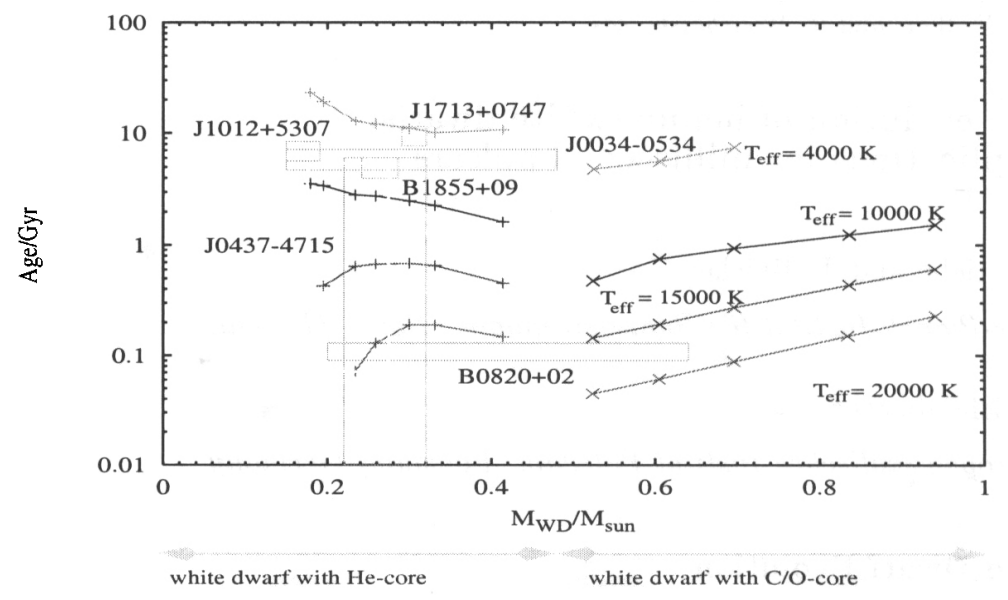

Figure 1. Ages of 6 MSP systems based on pulsar measurements as a function of the WD companion mass (see Burderi et al. 1998). The boxes refer to the uncertainties of spin-down ages and mass determinations. Cooling ages for evolutionary He- and C/O-WD models (Driebe et al. 1998, Blöcker 1995) are given for four effective temperatures.

et al. 1998, priv. comm.) gives a pulsar mass $M_{\text {pulsar }}=1.43 \pm 0.25 \mathrm{M}_{\odot}$ and $1.81 \pm 0.25 \mathrm{M}_{\odot}$, resp. From our models we derived a white dwarf age of $\approx 6 \mathrm{Gyr}$ in excellent agreement with the pulsar's spin-down age of 7 Gyr.

We studied other MSP systems as well assuming the correspondence of white dwarf cooling age and $\tau_{\text {spin-down. Selecting only systems with well given ages }}$ and/or masses, the white dwarf effective temperatures and surface gravities can be determined with the present evolutionary models. Figure 1 shows the results for 6 MSP systems. Note that the consideration of fully evolutionary He-WD models is crucial for the determination of the effective temperature. More details will be given in Schönberner et al. (1999).

\section{References}

Blöcker, T. 1995, A\&A 297, 727

Blöcker, T. et al. 1997, in: White Dwarfs,

J. Isern, M. Hernanz, E. Garcia-Berro (eds.), Kluwer, Dordrecht, p. 57

Burderi, L., King, A. R., Wynn, G. A. 1998, MNRAS 300, 1127

Callanan, P. J., Garnavich, P. M., Koester, D. 1998, MNRAS 298, 207

Driebe, T., Schönberner, D., Blöcker, T., Herwig, F. 1998, A\&A 339, 123

Driebe, T., Blöcker, T., Schönberner, D., Herwig, F. 1999, A\&A 350, 89

van Kerkwijk, M. H., Bergeron, P., Kulkarni, S. R. 1996, ApJ 467, L89

Nicastro, L. et al. 1995, MNRAS 273, L68

Sarna, M. J., Antipova, J., Muslimov, A. 1998, ApJ 499, 407

Schönberner, D., Driebe, T., Blöcker, T., 1999, A\&A, submitted 